\title{
Effects of UV-B radiation on development and hsp70 expression in sea urchin cleavage embryos
}

\author{
Rosa Bonaventura • Veronica Poma • \\ Roberta Russo - Francesca Zito - Valeria Matranga
}

Published online: 13 December 2006

(C) Springer-Verlag 2006

Erratum to: Mar Biol 149:79-86

DOI 10.1007/s00227-005-0213-0

In the pdf online version and in the print version, Fig. 4 appears twice, as Fig. 4 and as Fig. 5. The correct Fig. 5 is shown below.

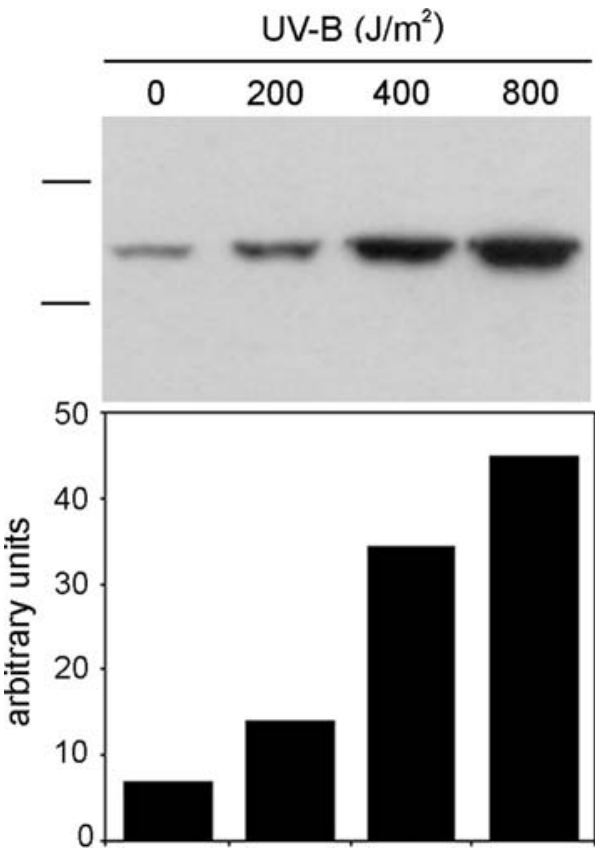

The online version of the original article can be found at http://dx.doi.org/ DOI 10.1007/s00227-005-0213-0.

R. Bonaventura $\cdot$ V. Poma $\cdot$ R. Russo $\cdot$ F. Zito .

V. Matranga $(\square)$

Consiglio Nazionale delle Ricerche,

Istituto di Biomedicina e Immunologia Molecolare

"Alberto Monroy", Palermo, Italy

e-mail: matranga@ibim.cnr.it

Fig. 5 Analysis of hsp70 protein levels in embryos exposed to high doses of UV-B. Western blot of total lysates from unexposed $\left(0 \mathrm{~J} / \mathrm{m}^{2}\right)$ and exposed $\left(200,400\right.$ and $\left.800 \mathrm{~J} / \mathrm{m}^{2}\right)$ embryos harvested $48 \mathrm{~h}$ after irradiation, and reacted with antihsp70. The positions of the molecular markers (97 and $66 \mathrm{kDa}$ ) are indicated on the left. The histogram shows result obtained by densitometric scannings of the filter, band intensities are reported in arbitrary units 